\title{
Femoral Nerve Pulsy due to Iliopsoas Muscle Hematoma: A Mini Literature Review
}

\section{Taisei Sako, Yuichirou Yokoyama, Keiji Hasegawa, Shintaro Tsuge, Kazumasa Nakamura, Akihito Wada, Hiroshi Takahashi}

Department of Orthopaedic Surgery, Toho University School of Medicine, Tokyo, Japan

Email:drkan@med.toho-u.ac.jp

How to cite this paper: Sako, T., Yokoyama, Y., Hasegawa, K., Tsuge, S., Nakamura, K., Wada, A. and Takahashi, H. (2017) Femoral Nerve Pulsy due to Iliopsoas Muscle Hematoma: A Mini Literature Review. Open Journal of Orthopedics, 7, 375-381. https://doi.org/10.4236/ojo.2017.711039

Received: August 25, 2017

Accepted: November 21, 2017

Published: November 24, 2017

Copyright $\odot 2017$ by authors and Scientific Research Publishing Inc. This work is licensed under the Creative Commons Attribution International License (CC BY 4.0).

http://creativecommons.org/licenses/by/4.0/

\begin{abstract}
We encountered a patient who developed femoral nerve paralysis due to iliopsoas muscle hematoma. Surgical removal of the hematoma is recommended for such cases, but conservative treatment may also be sufficient and there are no clear judgment criteria. An investigation of past reports showed that recovery was faster after surgery than after conservative treatment, regardless of the severity of paralysis, and that hematoma removal was especially effective for cases with severe paralysis. In our case, pain of the femoral nerve-innervated region was rapidly relieved by removal of the hematoma.
\end{abstract}

\section{Keywords}

Femoral Nerve Pulsy, Iliopsoas Hematoma, Therapy

\section{Introduction}

Cases of iliopsoas muscle hematoma are roughly divided into those caused by hemorrhagic diathesis, such as hemophilia and anticoagulant therapy, and those due to trauma [1] [2]. We encountered a patient with femoral nerve paralysis induced by iliopsoas muscle hematoma that formed during anticoagulant therapy for disseminated intravascular coagulation (DIC).

\section{Case Report}

The patient was a 69-year-old man with a chief complaint of right lower abdominal pain and right lower-extremity weakness. He was admitted to the Urology Department of our hospital for acute prostatitis-induced sepsis, and anticoagulant therapy with thrombomodulin for sepsis-induced DIC was initiated on the 4th hospital day. The patient felt pain in the right lower abdominal region over the anterior surface of the thigh during stepping on stairs on the 9th hospital day 
and became unable to extend the right hip joint due to pain on the 10th hospital day. Since right lower-extremity weakness was observed, the patient was referred to our department. His past medical history and history of oral medication were unremarkable.

Physical findings on the first examination included spontaneous pain in the right lower abdominal region over the right anterior surface of the thigh, and tense swelling in the right inguinal region. The hip joint showed a so-called psoas position in flexion, and adopting a supine position was difficult. Anesthesia of the right anterior surface of the thigh was noted. Muscle strength based on manual muscle testing (MMT) had scores of 2 for the right iliopsoas muscle and 0 - 1 for the right quadriceps muscle, and tendon reflex of the right patella was lost.

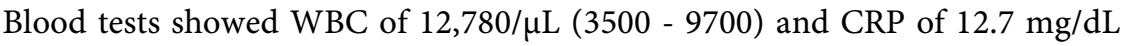
$(0-0.3)$, indicating the presence of an inflammatory reaction, and Plt was decreased to $9.6 \times 10^{4} \mu \mathrm{L}(14-37.9)$, meeting the acute-phase DIC score in diagnostic criteria [3].

In imaging, contrast-enhanced CT showed a slightly high-density space-occupying lesion in the right iliac fossa over the insertion site of the lesser trochanter in the iliac muscle, and the iliac muscle was swollen. There was no contrast enhancement inside or at the margin of the lesion (Figure 1).

Based on the acute development and imaging findings described above, femoral nerve paralysis induced by iliopsoas muscle hematoma, mainly in the iliac muscle, was suspected, and anticoagulant therapy was discontinued after the first examination at our department. The conditions of anesthesia and motor paralysis did not change, and $\mathrm{Hb}$ decreased from 12.8 to $6.0 \mathrm{~g} / 100 \mathrm{~mL}$ on the 12th hospital day. Since improvement of symptoms was not expected, the patient was transferred to a higher-level medical center on the 15th hospital day and invasive removal of the hematoma was performed on the same day.

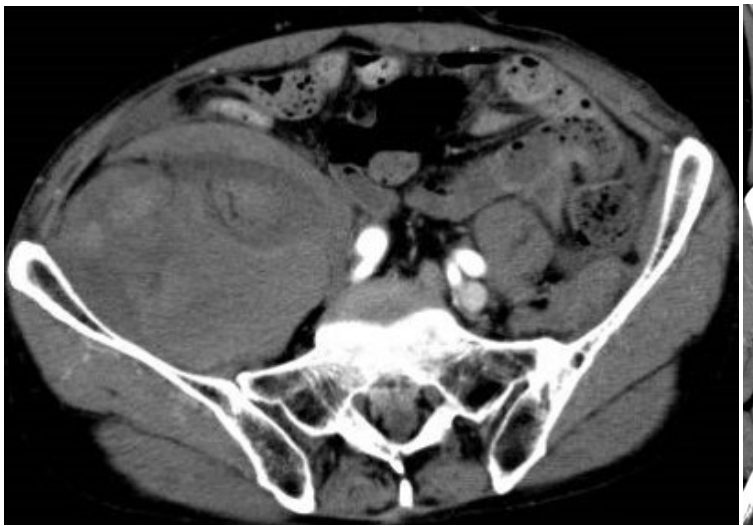

(a)

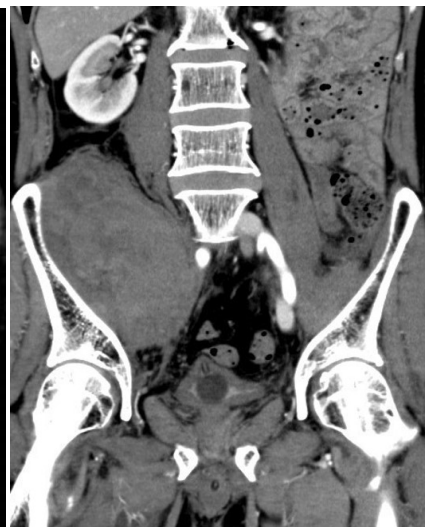

(b)

Figure 1. Contrast-enhanced CT showed a space-occupying lesion in the iliac fossa over the insertion site of the lesser trochanter in the iliac muscle in the right pelvic cavity, and the iliac muscle was markedly swollen. There was no contrast enhancement in the lesion or at the margin. (a) Axial view; (b) Coronal view. 
In surgery, the affected region was reached through a retroperitoneal approach in the left lateral position. The iliac muscle was markedly swollen and excluded the femoral nerve and psoas major muscle. Fasciotomy was applied to the iliac muscle, and a hematoma with a total weight of $150 \mathrm{~g}$ was removed (Figure 2 ), followed by confirmation of release of compression of the psoas major (Figure 3). After sufficient irrigation with saline, a drain was placed and surgery was completed by wound closure.

Right lower abdominal pain resolved immediately after surgery and the patient became able to extend the hip joint and adopt a supine position. Ambulation using a wheelchair was achieved on the day after surgery and the patient started walking training 5 days after surgery. He became able to walk independently with a cane 14 days after surgery and continued walking training and muscle strengthening exercise after discharge. Hematoma had disappeared on CT performed 3 months after surgery (Figure 4), and muscle strength at 6 months after surgery had a MMT score of 5 for the quadriceps muscle, showing improvement with slight remaining dysesthesia.

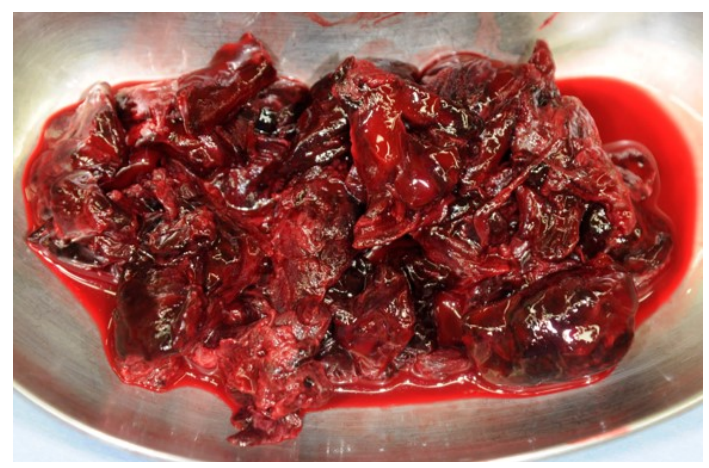

Figure 2. The iliac muscle was markedly swollen and a large blood clot (weight $150 \mathrm{~g}$ ) was excised when the fascia was manually incised.

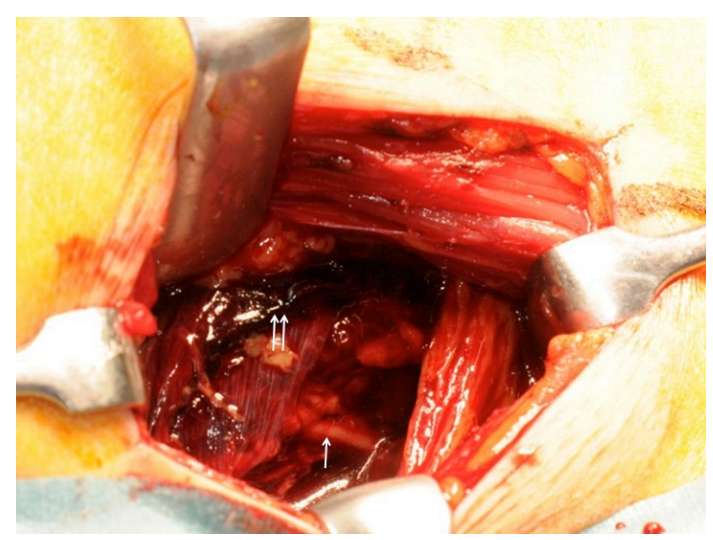

Figure 3. The hematoma was removed by fasciotomy of the iliac muscle, after which the iliac muscle shrunk $(\leftarrow \leftarrow)$ and the femoral nerve $(\leftarrow)$ was present right above the psoas major muscle. 


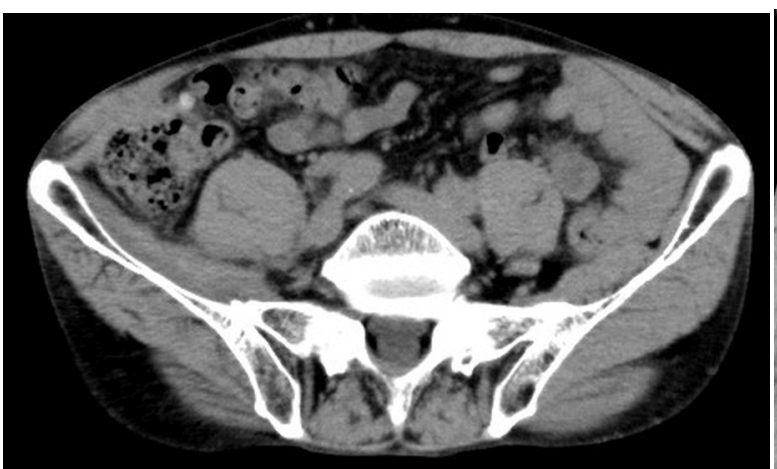

(a)

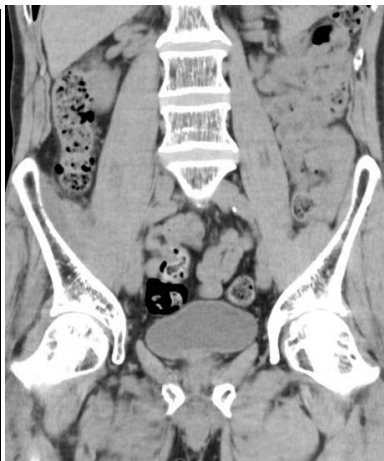

(b)

Figure 4. Hematoma had disappeared on CT performed 3 months after surgery, and muscle strength at 6 months after surgery had a MMT score of 5 for the quadriceps muscle, showing improvement with slight remaining dysesthesia. (a) Axial view; (b) Coronal view.

\section{Discussion}

The incidence of femoral nerve paralysis associated with iliopsoas muscle hematoma is relatively high, at about $70 \%$. When lower abdominal pain and femoral nerve paralysis develop acutely, iliopsoas muscle hematoma-induced femoral nerve paralysis should be closely examined. The anatomical factor in this condition may be the location of the femoral nerve in the space surrounded by the pelvic wall, inguinal ligament, and fascia of the iliac muscle and psoas major muscle, and swelling of the iliopsoas muscle due to hematoma may compress the nerve and cause paralysis [4].

Favorable recovery from paralysis by removal of the hematoma [5] [6] [7] [8] and rapid pain relief by surgery [5] [9] have been reported in treatment of iliopsoas muscle hematoma accompanied by femoral nerve paralysis. In contrast, another study reported that the efficacy of surgery was unclear because the function of the quadriceps muscle could be recovered by conservative treatment [1]; therefore, there are no clear criteria for a surgical indication at present. CT-guided needle aspiration of hematoma has also been reported [10], but the hematoma is hard and organized in many cases, for which needle aspiration is not indicated.

For these reasons, we investigated the outcomes of conservative and surgical treatments of iliopsoas muscle hematoma accompanied by femoral nerve paralysis. A literature search revealed reports of 28 cases of iliopsoas muscle hematoma accompanied by femoral nerve paralysis [1] [2] [4]-[24]. The causes were hemorrhagic diathesis, such as anticoagulant therapy, hemophilia, and thrombolytic therapy in 13 cases, while 15 cases were associated with exercise, trauma, and hip joint disease. In our patient, anticoagulant therapy for DIC was considered to be the cause. The treatment method was conservative treatment in 8 cases and hematoma removal in 20 cases. The time to resolution of symptoms was described in 24 of the 28 cases, and was unclear in 4 . Muscle strength recovered to MMT 4 or higher in 23 cases, while muscle weakness of MMT 3 re- 
mained in one case, which received conservative treatment [6]. The times to resolution of mild hypesthesia and recovery of lower limb muscle strength to MMT 4 or higher were 3.6 and 2.3 months in cases treated with conservative and surgical treatment, respectively. This suggests that recovery is faster after surgical treatment and that paralysis may persist with conservative treatment.

Investigation of the surgical indication may be necessary depending on the ADL level and grade of paralysis in individual patients because complications may occur, such as hemorrhage, iatrogenic nerve injury, and postoperative infection [5]. However, the incidence of these complications is unlikely to be high, and rapid pain relief by hematoma removal and shortening of the time to recovery from paralysis may be greater benefits for patients. In our patient with iliopsoas muscle hematoma accompanied by severe femoral nerve paralysis, rapid pain relief and early recovery from paralysis were achieved by hematoma removal, and the degree of satisfaction of the patient with relief from pain after surgery was very high. Therefore, we suggest that surgical treatment should be selected for patients with this condition with severe paralysis who do not desire conservative treatment.

\section{Conclusion}

We encountered a patient who developed femoral nerve paralysis induced by iliopsoas muscle hematoma during anticoagulant therapy. Rapid pain relief and early improvement of femoral nerve paralysis were achieved by surgical treatment.

\section{Disclosure}

Consent to publication of photographs and data were given by the patient and his family after they received an explanation of this process. There is no potential conflict of interest to disclose with regard to this case.

\section{References}

[1] Patel, A., Calfee, R., Thakur, N. and Eberson, C. (2008) Non-Operative Management of Femoral Neuropathy Secondary to a Traumatic Iliacushaematoma in an Adolescent. The Journal of Bone and Joint Surgery, 90, 1380-1381. https://doi.org/10.1302/0301-620X.90B10.21040

[2] Pirouzmand, F. and Midha, R. (2001) Subacute Femoral Compressive Neuropathy from Iliacus Compartment Hematoma. Canadian Journal of Neurological Sciences, 28, 155-158. https://doi.org/10.1017/S0317167100052860

[3] Asakura, H., Takahashi, H., Uchiyama, T., Eguchi, Y., Okamoto, K., Kawasugi, K., Madoiwa, S. and Wada, H. (2016) Proposal for New Diagnostic Criteria for DIC from the Japanese Society on Thrombosis and Hemostasis. Thrombosis Journal, 14, 42. https://doi.org/10.1186/s12959-016-0117-x

[4] Goodfellow, J., Fearn, C.B. and Matthews, J.M. (1967) Iliacus haematoma. A Common Complication of Haemophilia. The Journal of Bone and Joint Surgery, 49, 748-756. 
[5] Qian, J., Jing, J.H., Tian, D.S., Zhang, J.S. and Chen, L. (2014) Safety and Efficacy of a New Procedure for Treating Traumatic Iliopsoas Hematoma: A Retroperitoneoscopic Approach. Surgical Endoscopy, 28, 265-270. https://doi.org/10.1007/s00464-013-3183-1

[6] Basheer, A., Jain, R., Anton, T. and Rock, J. (2013) Bilateral Iliopsoas Hematoma: Case Report and Literature Review. Surgical Neurology International, 4, 121. https://doi.org/10.4103/2152-7806.118561

[7] Jahollari, A., Cavolli, R., Tavlasoglu, M., Sallahu, F. and Muriqi, S. (2013) Iliopsoas Hematoma Due to Muscular Rupture Following Defibrillation. Ulusal Travma ve Acil Cerrahi Dergisi, 19, 564-566. https://doi.org/10.5505/tjtes.2013.74152

[8] Ganu, S. and Mehta, Y. (2013) Femoral Compressive Neuropathy from Iliopsoas Haematoma Complicating Dengue Hemorrhagic Fever. Asian Pacific Journal of Tropical Medicine, 6, 419-420. https://doi.org/10.1016/S1995-7645(13)60052-8

[9] Kumar, S. and Pflueger, G. (2016) Delayed Femoral Nerve Palsy Associated with Iliopsoas Hematoma after Primary Total Hip Arthroplasty. Case Reports in Orthopedics, 2016, Article ID: 6963542. https://doi.org/10.1155/2016/6963542

[10] Holscher, R.S., Leyten, F.S., Oudenhoven, L.F. and Puylaert, J.B. (1997) Percutaneous Decompression of an Iliopsoas Hematoma. Abdominal Imaging, 22, 114-116. https://doi.org/10.1007/s002619900154

[11] Podger, H. and Kent, M. (2016) Femoral Nerve Palsy Associated with Bilateral Spontaneous Iliopsoas Haematomas: A Complication of Venous Thromboembolism Therapy. Age Ageing, 45, 175-176. https://doi.org/10.1093/ageing/afv176

[12] Niakan, E., Carbone, J.E., Adams, M. and Schroeder, F.M. (1991) Anticoagulants, Iliopsoas Hematoma and Femoral Nerve Compression. American Family Physician, 44, 2100-2102.

[13] Bapat, V.N., Agrawal, N.B. and Tendolkar, A.G. (1996) Femoral Nerve Palsy: A Rare Presentation of Femoral Artery Pseudoaneurysm Following Cardiac Catheterisation. Indian Heart Journal, 48, 715-716.

[14] Nakao, A., Sakagami, K., Mitsuoka, S., Uda, M. and Tanaka, N. (2001) Retroperitoneal Hematoma Associated with Femoral Neuropathy: A Complication under Antiplatelets Therapy. Acta Med Okayama, 55, 363-366.

[15] Wada, Y., Yanagihara, C. and Nishimura, Y. (2005) Bilateral Iliopsoas Hematomas Complicating Anticoagulant Therapy. Internal Medicine, 44, 641-643. https://doi.org/10.2169/internalmedicine.44.641

[16] Fernandes, C., Pereira, P. and Rodrigues, M. (2015) Spontaneous Iliopsoas Muscle Haematoma as a Complication of Anticoagulation in Acute Cerebral Venous Thrombosis: to Stop or Not to Stop (The Anticoagulation)? BMJ Case Reports, bcr2014206410. https://doi.org/10.1136/bcr-2014-206410

[17] Freeman, A. (2015) Acquired Haemophilia A Presenting at a District General Hospital. BMJ Case Reports, bcr2014208001. https://doi.org/10.1136/bcr-2014-208001

[18] Spengos, K., Anagnostou, E. and Vassilopoulou, S. (2012) Subacute Proximal Leg Weakness after a Minor Traffic Accident in a Patient Treated with Anticoagulants. BMJ Case Reports, 0220125731.

[19] Selton, J., Perrin, J., Ropion, H., Abdelfatah, M., Siouala, B., Pruna, L. and Kaminsky, P. (2011) Iliopsoas Hematoma in Gaucher's Disease. Internal Medicine, 50, 2643-2647. https://doi.org/10.2169/internalmedicine.50.5215

[20] Keikhaei, B. and SoltaniShirazi, A. (2011) Spontaneous Iliopsoas Muscle Hematoma in a Patient with von Willebrand Disease: A Case Report. Journal of Medical Case 
Reports, 5, 274. https://doi.org/10.1186/1752-1947-5-274

[21] Tokarz, V.A., McGrory, J.E., Stewart, J.D. and Croslin, A.R. (2003) Femoral Neuropathy and Iliopsoas Hematoma as a Result of Postpartum Factor-VIII Inhibitor Syndrome. A Case Report. The Journal of Bone and Joint Surgery, 85, 1812-1815. https://doi.org/10.2106/00004623-200309000-00025

[22] Fernandez-Palazzi, F., Hernandez, S.R., De Bosch, N.B. and De Saez, A.R. (1996) Hematomas within the Iliopsoas Muscles in Hemophilic Patients: The Latin American Experience. Clinical Orthopaedics and Related Research, 328, 19-24. https://doi.org/10.1097/00003086-199607000-00005

[23] King, R.B. and Bechtold, D.L. (1985) Warfarin-Induced Iliopsoas Hemorrhage with Subsequent Femoral Nerve Palsy. Annals of Emergency Medicine, 14, 362-364. https://doi.org/10.1016/S0196-0644(85)80106-2

[24] Ho, K.J., Gawley, S.D. and Young, M.R. (2003) Psoas Haematoma and Femoral Neuropathy Associated with Enoxaparin Therapy. International Journal of Clinical Practice, 57, 553-554. 